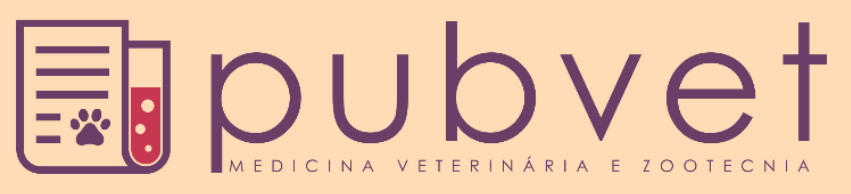

ISSN $1982-1263$

https://doi.org/10.31533/pubvet.v13n3a284.1-5

\title{
Mandibulectomia parcial em cão com melanoma: Relato de caso
}

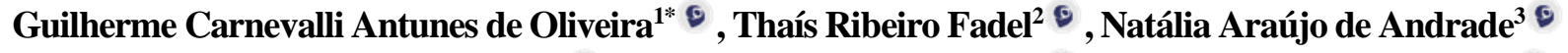 \\ Emanuelle Araújo Nunes Carneiro $^{4}$, Moacir Santos de Lacerda ${ }^{\circ}$, Renato Linhares Sampaio ${ }^{5}$ \\ ${ }^{I}$ Aprimorando anestesiologia veterinária pela Universidade de Uberaba, Uberaba-MG, Brasil. \\ ${ }^{2}$ Mestranda USP Pirassununga, Pirassununga - SP, Brasil \\ ${ }^{3}$ Veterinária autônoma, Boa Esperança - MG, Brasil \\ ${ }^{4}$ Preceptora de clínica de pequenos animais Universidade de Uberaba, Uberaba-MG, Brasil \\ ${ }^{5}$ Professor na Universidade de Uberaba, Uberaba-MG, Brasil \\ *Autor para correspondência, E-mail: anestesia.veterinaria@outlook.com
}

Resumo. Os tumores melanocíticos se originam dos melanócitos e melanoblastos, sendo que a conversão dos melanócitos normais em neoplásicos é constituída de múltiplas etapas. Melanomas são neoplasias cutâneas primárias encontradas em regiões que possuem acúmulo de melanóticos. O melanoma é formado por uma mácula escura que se transforma em uma massa firme, de crescimento rápido com infiltração local e metástase precoce. $\mathrm{O}$ tamanho e o grau de pigmentação não são parâmetros indicadores de malignidade tumoral. As neoplasias da cavidade oral em cães e gatos são frequentes e representam o quarto local mais acometido por tumores, sendo o melanoma o mais frequente. O diagnóstico baseia-se no histopatológico e a excisão cirúrgica continua sendo método de tratamento mais indicado. A mandibulectomia total ou parcial aumenta a sobrevida do animal em até 10 meses, sem associação de qualquer terapia adjuvante. Se não houver a retirada com margem do tumor, a sobrevida é de 3 a 4 meses. Objetivou-se relatar um caso clínico de uma cadela, da raça poodle com 14 anos de idade, que apresentou um nódulo em região rostral da mandíbula, com crescimento rápido, na qual foi diagnosticada com melanoma e optou-se pela mandibulectomia parcial, notado melhor qualidade de vida ao paciente sem apresentar uma condição estética desagradável ao proprietário.

Palavras chave: câncer, melanócitos, neoplasias, malignidade, infiltrativo

\section{Partial mandibulectomy in dog with melanoma: Case report}

Abstract. Melanocytic tumors are originated from melanocytes and melanoblasts, the conversion of normal melanocytes into neoplasms consists of multiple stages. Melanomas are primary cutaneous neoplasms found at body regions that have accumulation of melanocytes. Melanoma is formed by a dark macula that turns into a firm, fast-growing mass with local infiltration and early metastasis. The size and degree of pigmentation are not indicators of tumor malignancy. Neoplasms of the oral cavity in dogs and cats are frequent and represent the fourth site most affected by tumors, which melanoma being the most frequent. The diagnosis is based on histopathology and surgical excision remains the most indicated method of treatment. Total or partial mandibulectomy increases the survival of the animal within 10 months without any combination of adjuvant therapy. if it's not removed with tumor security margin, the survival increases 3 to 4 months. The objective of this study is to report a clinical case of a 14-year-old poodle dog with a nodule at the rostral region of the jawbone, with fast growth, in which the patient was diagnosed with melanoma in which 
partial mandibulectomy showed a better quality of life quality of life in this case, without having an unpleasant $g$ an unpleasant aesthetic condition to the owner.

Key words: cancer, melanocytes, neoplasia, malignancy, infiltrative

\title{
Mandibulectomía parcial en perro con melanoma: Relato de caso
}

\begin{abstract}
Resumen. Los tumores melanocíticos se originan de los melanocitos y melanoblastos, siendo que la conversión de los melanocitos normales en neoplásicos está constituida por múltiples etapas. Melanomas son neoplasias cutáneas primarias encontradas en regiones que poseen la acumulación de melanocitos. El melanoma está formado por una mancha oscura que se transforma en una masa firme, de crecimiento rápido con infiltración local y metástasis precoz. El tamaño y el grado de pigmentación no son parámetros indicadores de malignidad tumoral. Las neoplasias de la cavidad oral en perros y gatos son frecuentes y representan el cuarto lugar más acometido por tumores, siendo el melanoma más frecuente. El diagnóstico se basa en el histopatológico y la escisión quirúrgica sigue siendo el método de tratamiento más indicado. La mandibulectomía total o parcial aumenta la supervivencia del animal en hasta 10 meses, sin asociación de ninguna terapia adyuvante. Si no hay retirada con margen del tumor, la supervivencia es de 3 a 4 meses. En el presente estudio se evaluó un caso clínico de una perra, de la raza Poodle con edad de 14 años, que presentó un nódulo en región rostral de la mandíbula, con crecimiento rápido, en la cual fue diagnosticada con melanoma e inmediatamente se optó por la mandibulectomía parcial, notadose mejor calidad de vida al paciente sin presentar una condición estética desagradable al propietario.
\end{abstract}

Palabras clave: cáncer, melanocitos, neoplasia, malignidad, infiltración

\section{Introdução}

Os tumores melanocíticos se originam dos melanócitos e melanoblastos (Garma-Aviña et al., 1981; Modiano et al., 1999), se manifestam em todos os animais domésticos e de laboratório (Conroy, 1967). A conversão de melanócitos normais em neoplásicos é um processo constituído por múltiplas etapas, tendo a iniciação como evento primário, seguida por promoção, transformação e metástase (Smith et al., 2002).

A terminologia das neoplasias melanocíticas nos animais domésticos não é homogênea. Alguns autores usam o termo melanoma cutâneo para descrever o tumor que demonstra diferenciação melanocítica, com potencial metastático não conhecido ou comprovado (Goldschmidt, 1994; Goldschmidt \& Shofer, 1992). Em contrapartida esses mesmos tumores têm sido chamados de melanocitoma por outros autores (Gross et al., 2008; Scott et al., 2001; Smith et al., 2002). Outros autores usam o termo melanoma maligno para indicar o tumor com potencial metastático conhecido ou comprovado (Goldschmidt, 1994; Goldschmidt \& Shofer, 1992). Neste estudo iremos utilizar o termo melanoma. Melanomas são neoplasias cutâneas primárias, não obstante podem ser encontradas em qualquer localização anatômica que possua acúmulo de melanóticos (Yager \& Scott, 1993). Estas neoplasias podem se apresentar na forma maligna ou benigna; porém, na maioria das vezes possuem prognóstico desfavorável. Elas podem ser encontradas na pele, cavidade oral, e nos olhos de cães, equinos e suínos. Usualmente se originam em locais em que as células precursoras são numerosas. (Coelho, 2016; Núñez \& Bouda, 2007). O melanoma é formado por uma mácula escura que se transforma em uma massa firme, de crescimento rápido com infiltração local e metástase precoce. $\mathrm{O}$ tamanho e o grau de pigmentação não são parâmetros indicadores de malignidade tumoral (Coelho, 2016; Goldschmidt \& Hendrick, 2002; Núñez \& Bouda, 2007).

Nos cavalos tordilhos, o melanoma é muito comum (Coelho, 2016; Goldschmidt \& Hendrick, 2002; Núñez \& Bouda, 2007). Nos felinos as neoplasias melanocíticas benignas e malignas são incomuns e ocorrem praticamente com a mesma frequência (Scott et al., 2001). Nos caninos, as neoplasias melanocíticas são comuns (Cotchin, 1959; Modiano et al., 1999; Scott et al., 2001), correspondendo de 4 a 10\% de todas as neoplasias cutâneas dessa espécie, sendo que menos de 55\% são malignas (Smith et al., 2002). Na espécie canina o melanoma é o tumor mais comum da cavidade oral (Bostock, 1979; Modiano 
et al., 1999; Smith et al., 2002) e dos olhos (Sullivan et al., 1996) e a segunda mais frequente dos dígitos com localização subungueal (Scott et al., 2001; Smith et al., 2002). As neoplasias da cavidade oral em cães e gatos são frequentes e representam o quarto local mais acometido por tumores, sendo o melanoma o mais frequente (Rodrígues-Queiróz et al., 1998). O diagnóstico baseia-se no histopatológico e a excisão cirúrgica continua sendo método de tratamento mais indicado (Verstraete, 2005).

As opções de tratamento para o melanoma, além da ressecção cirúrgica radical, incluem radioterapia (Banks \& Morris, 1975; Burk, 1996), fotocoagulação (Frimberger et al., 1998; Sullivan et al., 1996), quimioterapia (Sulaimon \& Kitchell, 2001), imunoterapia e geneterapia (Quintin-Colonna et al., 1996; Rochlitz et al., 1999; Vail \& Macewen, 2000). O animal deste relato passou apenas pela ressecção cirúrgica.

\section{Relato de caso}

Foi atendida no hospital veterinário de Uberaba, uma cadela com 14 anos de idade, da raça poodle, pesando $10,6 \mathrm{~kg}$, apresentando nódulo em região rostral da mandíbula, com crescimento rápido, aproximadamente uma semana. $\mathrm{O}$ animal encontrava-se inapetente, prostrado e com odor fétido oral. Foram realizados exames laboratoriais de hemograma, ureia, creatinina e ALT, também foi realizado exame radiográfico, todos dentro dos padrões de normalidade. Optou-se pela excisão cirúrgica com margem do nódulo como tratamento de eleição e foi realizado o exame histopatológico, o qual indicou que a neoplasia em questão se tratava de melanoma.

Como protocolo anestésico foi realizado morfina na dose de $0,5 \mathrm{mg} / \mathrm{kg}$ associada à Acepromazina na dose de $0,03 \mathrm{mg} / \mathrm{kg}$ como medicação pré-anestésica, cetamina $2 \mathrm{mg} / \mathrm{kg}$, associado ao Midazolam 0,3 $\mathrm{mg} / \mathrm{kg}$ e propofol $2 \mathrm{mg} / \mathrm{kg}$ para indução, e manutenção com isofluorano diluído no oxigênio a $100 \%$ pelo método de borbulhamento em sistema circular valvular. Foi realizado bloqueio anestésico local do nervo alveolar mandibular com lidocaína $2 \%$ na dose de $4 \mathrm{mg} / \mathrm{kg}$.

Procedeu-se à cirurgia, sendo realizada uma incisão gengival em torno da mandíbula até o primeiro pré-molar atrás dos caninos bilateralmente, posteriormente a osteotomia com serra manual atrás do $1^{\circ}$ pré-molar bilateralmente, foi então retirada a porção mandibular rostral com a realização de reconstrução oral utilizando a técnica de $\mathrm{V}$ plastia bilateral. A síntese de subcutâneo e gengiva foi feita com fio Vicryl $3-0$ e a pele com Nylon 3-0 com ambos os planos com pontos simples separado.

Sete meses e meio após o procedimento cirúrgico, notou-se que o nódulo recidivou, com tamanho aproximado de $0,4 \mathrm{~cm} \times 0,5 \mathrm{~cm}$ rostral e no antimero direito (Figura 1B). Passados 20 dias o nódulo evoluiu para aproximadamente $7 \times 6 \mathrm{~cm}$ (Figura 1C), expandindo para a gengiva esquerda, mal delimitada, infiltrativa, consistência firme e com várias áreas de ulcerações, o animal apresentava dor, inapetência e caquexia. Devido ao prognóstico reservado e impossibilidade de realizar outros tratamentos adjuvantes tais como quimioterapia e/ou radioterapia, fotocoagulação, imunoterapia foi optado pela eutanásia do animal.

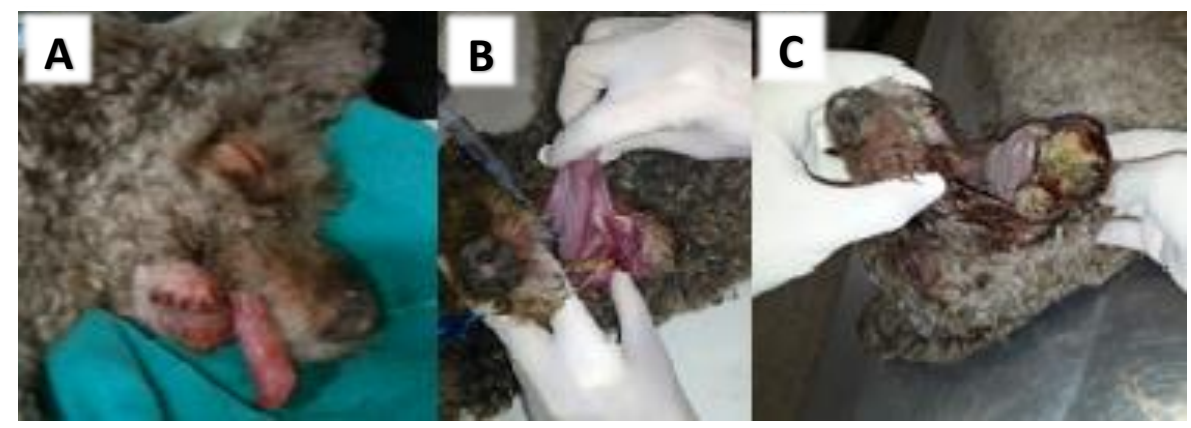

Figura 1. A: Pós cirúrgico imediato, B: 7 meses e meio depois, C: 8 meses depois.

\section{Discussão}

A mandibulectomia total ou parcial aumenta a sobrevida do animal em até 10 meses, sem associação de qualquer terapia adjuvante. Se não houver a retirada com margem do tumor, a sobrevida é de 3 a 4 meses (Proulx et al., 2002), apenas 10\% dos pacientes diagnosticados com melanoma maligno 
apresentam sobrevida maior que um ano (Birchard \& Sherding, 2008; Dyce et al., 2004; Sisson et al., 1986). Corroborando com o ocorrido com o cão, houve excelente recuperação pós-cirúrgica, sem dificuldades na apreensão do alimento, e apresentou um tempo de sobrevida de 7 meses até a recidiva. Uma alternativa para se aumentar o tempo de sobrevida, seria a associação da quimioterapia com a excisão cirúrgica radical, com ampla margem de segurança (cerca de 1 a $3 \mathrm{~cm}$ ), ou com a criocirurgia ou radioterapia, quando a ablação não puder ser completa (Bostock, 1979).

\section{Conclusões}

Este trabalho mostra a importância do tratamento cirúrgico com margem e boa anestesia, que pode melhorar a qualidade de vida do paciente sem apresentar uma condição estética desagradável ao proprietário. As técnicas cirúrgica e anestésica instauradas neste caso mostraram-se eficazes, pois o animal não apresentou dificuldades ou dor durante a alimentação e apresentou-se fisicamente agradável, contribuindo assim com a melhor qualidade de vida, e tempo de sobrevida de 8 meses para o paciente. No entanto, por ser uma neoplasia de alta malignidade e alto poder metastático, outras técnicas devem ser adicionadas, tais como radioterapia, quimioterapia, criocirurgia, imunoterapia, geneterapia, a fim de tentar proporcionar maior tempo de sobrevida ao paciente.

\section{Referências bibliográficas}

Banks, W. C. \& Morris, E. (1975). Results of radiation treatment of naturally occurring animal tumors [Dogs]. Journal American Veterinary Medical Association, 166(11):1063-1064.

Birchard, S. J. \& Sherding, R. G. (2008). Manual Saunders: clínica de pequenos animais (Vol. 3). São Paulo.

Bostock, D. E. (1979). Prognosis after surgical excision of canine melanomas. Veterinary Pathology, 16(1):32-40.

Burk, R. L. (1996). Radiation therapy in the treatment of oral neoplasia. Veterinary Clinics: Small Animal Practice, 26(1):155-163.

Coelho, H. E. (2016). Patologia veterinária. Uberaba, Minas Gerais: Coelho.

Conroy, J. D. (1967). Melanocytic tumors of domestic animals: With special reference to dogs. Archives of Dermatology, 96(4):372-380.

Cotchin, E. (1959). Some tumours of dogs and cats of comparative veterinary and human interest. The Veterinary Record, 71(45):1040-1050.

Dyce, K. M., Wensing, C. J. G. \& Sack, W. O. (2004). Tratado de anatomia veterinária. São Paulo: Elsevier Brasil.

Frimberger, A. E., Moore, A. S., Cincotta, L., Cotter, S. M. \& Foley, J. W. (1998). Photodynamic therapy of naturally occurring tumors in animals using a novel benzophenothiazine photosensitizer. Clinical Cancer Research, 4(9):2207-2218.

Garma-Aviña, A., Valli, V. E. \& Lumsden, J. H. (1981). Cutaneous melanomas in domestic animals. Journal of Cutaneous Pathology, 8(1):3-24.

Goldschmidt, M. H. (1994). Pigmented lesions of the skin. Clinics in Dermatology, 12(4):507-514.

Goldschmidt, M. H. \& Hendrick, M. J. (2002). Tumors of the skin and soft tissues. In D. J. Meuten (Ed.), Tumors in domestic animals (pp. 45-117). Iowa, USA: Iowa State Press.

Goldschmidt, M. H. \& Shofer, F. S. (1992). Skin tumors of the dog and cat. Oxford: Pergamon Press Ltd.

Gross, T. L., Ihrke, P. J., Walder, E. J. \& Affolter, V. K. (2008). Skin diseases of the dog and cat: clinical and histopathologic diagnosis. Oxford: John Wiley \& Sons.

Modiano, J. F., Ritt, M. G. \& Wojcieszyn, J. (1999). The molecular basis of canine melanoma: pathogenesis and trends in diagnosis and therapy. Journal of Veterinary Internal Medicine, 13(3):163-174.

Núñez, O. L. \& Bouda, J. (2007). Patología Clínica Veterinaria (Vol. 1. ed). Mexico: FMVZ-UNAM. 
Proulx, K., Richard, D. \& Walker, C.-D. (2002). Leptin regulates appetite-related neuropeptides in the hypothalamus of developing rats without affecting food intake. Endocrinology, 143(12):4683-4692.

Quintin-Colonna, F., Devauchelle, P., Fradelizi, D., Mourot, B., Faure, T., Kourilsky, P., . . Mehtali, M. (1996). Gene therapy of spontaneous canine melanoma and feline fibrosarcoma by intratumoral administration of histoincompatible cells expressing human interleukin-2. Gene Therapy, 3(12):1104-1112.

Rochlitz, C., Jantscheff, P., Bongartz, G., Dietrich, P.-Y., Quiquerez, A. L., Schatz, C., . . Dorval, T. (1999). Gene therapy study of cytokine-transfected xenogeneic cells (Vero-interleukin-2) in patients with metastatic solid tumors. Cancer Gene Therapy, 6(3):271-281.

Rodrígues-Queiróz, J., Trobo Muñiz, J. \& San Román, F. (1998). Neoplasias orales en pequeños animales. Cirurgía maxilofacial I. In F. S. Roman (Ed.), Atlas de Odontología en Pequeños Animales (Vol. 1). Madrid, Espanha: Grass Edicions.

Scott, D. W., H., M. W. \& E., G. C. (2001). Mullerand Kirk's small animal dermatology. Philadelphia, USA: WB. Saunders.

Sisson, S., Grossman, J. D. \& Getty, R. (1986). Anatomia dos animais domésticos. Rio de Janeiro: Interamericana.

Smith, S. H., Goldschmidt, M. H. \& McManus, P. M. (2002). A comparative review of melanocytic neoplasms. Veterinary Pathology, 39(6):651-678.

Sulaimon, S. S. \& Kitchell, B. E. (2001). Canine melanoma: combating a destructive and deadly tumor. Veterinary Medicine, 96(12):931-942.

Sullivan, T. C., Nasisse, M. P., Davidson, M. G. \& Glover, T. L. (1996). Photocoagulation of limbal melanoma in dogs and cats: 15 cases (1989-1993). Journal of the American Veterinary Medical Association, 208(6):891-894.

Vail, D. M. \& Macewen, E. G. (2000). Spontaneously occurring tumors of companion animals as models for human cancer. Cancer Investigation, 18(8):781-792.

Verstraete, F. J. M. (2005). Mandibulectomy and maxillectomy. Veterinary Clinics of North America: Small Animal Practice, 35(4):1009-1039.

Yager, J. A. \& Scott, D. W. (1993). The skin and appendages. In K. V. C. Jubb \& P. C. Kennedy (Eds.), The pathology of domestic animals (Vol. 1, pp. 531-738). San Diego, USA: Academic Press.

Recebido: 13 de fevereiro, 2019.

Aprovado: 2 de março, 2019.

Publicado: 19 de março, 2019.

Licenciamento: Este artigo é publicado na modalidade Acesso Aberto sob a licença Creative Commons Atribuição 4.0 (CC-BY 4.0), a qual permite uso irrestrito, distribuição, reprodução em qualquer meio, desde que o autor e a fonte sejam devidamente creditados. 